\title{
潾
}

\section{UNE RELECTURE D’EDWARD SAID}

Resumé: L'article propose une relecture de l'ouvrage principal de Said, qui a eu une forte influence sur la géographie au cours des dernières décennies. L'orientalisme, selon Said, est un discours sur l'Orient, une construction mentale qui légitime le processus de la domination européenne sur les territoires colonisés. L'accent est mis sur l'importance de l'œuvre de Said, qui met en évidence la construction historique de ces entités géographiques que sont l'Est et l'Ouest. Le texte souligne également quelques faiblesses ou les limites de l'interprétation des idées de Said sur l'orientalisme. Edward Said néglige la diversité des images que l'orientalisme construit et ne s'interrogue pas sur les contradictions et les problèmes de l'Occident lui-même. Il est important de voir qu'il n'y a pas un seul Orient ou um seul orientalisme, mais une diversité d'orientalismes. La construction et la compréhension des relations entre l'Est et I'Ouest peut resulted de plusieurs geographies imaginaires.

Mots-clés: Edward Said; Orientalisme; Géographies imaginaires

Depuis sa publication en 1978 (1980 en France), l'Orientalisme d'Edward Said a fortement marqué la géographie ; son influence s'est d'abord affirmée dans les pays de langue anglaise, où elle a inspiré le courant postcolonial; en France, elle est sensible depuis une quinzaine d'années. Pourquoi cet engouement de notre discipline pour le travail d'un spécialiste de littérature comparée?

L'Orientalisme retrace l'histoire d'une brillante discipline académique. Sous 
sa forme universitaire, elle se développe au début du $\mathrm{XIX}^{\mathrm{e}}$ siècle, mais a ses racines dans la culture classique - François I ${ }^{\text {er }}$ n'a-t-il pas créé le Collège de France pour que le pays dispose d'un enseignement de langues orientales que ne dispensait pas la Sorbonne? Pour Said, la généalogie de l'orientalisme est plus ancienne encore - ses racines se situent dans l'Antiquité grecque, chez Eschyle ou chez Euripide par exemple.

\section{La thèse d'Edward Said: De l'analyse au discours et à la domination La dimension géographique}

L'orientalisme s'attache à un objet géographique: il étudie les peuples, les cultures, les religions, les textes et les monuments qui se situent à l'Est de l'Europe, dans le Proche- et le MoyenOrient, et au-delà, en Inde, en Chine, au Japon. Est-ce un ensemble territorial dont les contours s'imposent d'eux-mêmes à l'observateur? Non :

\begin{abstract}
prendre au sérieux l'observation de Vico: les hommes font leur propre histoire, ce qu'ils peuvent connaître, c'est ce qu'ils ont fait - et l'appliquer aussi à la géographie : en tant qu'entités géographiques et culturelles à la fois - sans parler d'entités historiques des lieux, des régions, des secteurs géographiques tels que 'l'Orient' et 'l'Occident' ont été fabriqués par l'homme. C'est pourquoi, tout autant que l'Occident lui-même, l'Orient est une idée qui a une histoire et une tradition de pensée, une imagerie et un vocabulaire qui lui ont donné une réalité et une présence en Occident et pour l'Occident. Les deux entités géographiques se soutiennent ainsi et, dans une certaine mesure, se reflètent l'une l'autre" (Said, 1980/1978, p. 17).
\end{abstract}

"J'ai commencé par faire l'hypothèse que l'Orient n'est pas un fait de nature inerte. Il n'est pas simplement là, tout comme l'Occident n'est pas non plus simplement là. Nous devons
Dans les années 1970, les géographes ne considéraient plus la plupart des entités territoriales qu'ils étudiaient comme des ensembles naturels; c'étaient des constructions humaines, ils l'avaient 
compris. Lorsqu'ils analysaient ces réalisations, ils se montraient cependant plus attentifs au travail des hommes, à la mise en place d'équipements et d'infrastructures, à la structuration de réseaux urbains, à la maîtrise des milieux et à l'exploitation des mines qu'aux images mentales qu'en avaient les différents acteurs. On savait, depuis Giraud-Soulavie, à la fin du XVIII ${ }^{e}$ siècle, que les divisions régionales existaient aussi dans l'esprit des gens - qui en parlaient comme de pays -, mais c'est avec une certaine réticence que les géographes abordaient ces réalités. Edward Said n'a pas ces timidités : l'Orient dont il traite est une construction mentale; à la différence de celles auxquelles les géographes s'étaient jusque-là arrêtés, celleci ne repose pas sur la perception de la réalité locale par les gens qui y vivent et qui la vivent ; elle est découpée de l'extérieur, par des spécialistes qui la projettent sur une portion d'espace dont au départ, personne autour d'eux n'a d'expérience directe.

L'audace de Said réside aussi dans les dimensions de l'aire qu'il analyse: les géographes raisonnaient généralement sur de petits ensembles. L'Orient de Said est flou, ses limites varient selon les époques, mais il est immense. Il a bien sûr une réalité matérielle, géographique au sens habituel du terme :

\begin{abstract}
"La géographie était, pour l'essentiel, le matériau de soutènement de la connaissance sur l'Orient. Toutes les caractéristiques latentes et constantes de l'Orient reposaient sur sa géographie, y étaient enracinées. Ainsi, d'une part, l'Orient géographique nourrissait ses habitants, garantissait leurs caractères propres et définissait leur spécificité ; de l'autre, l'Orient géographique sollicitait l'attention de l'Occident [...]" (Said, 1980/1978, p. 247).
\end{abstract}

C'est sur cette sollicitation que porte l'attention de Said. Il ne s'attache pas aux caractéristiques géographiques, culturelles et sociales de l'Orient, mais à la manière dont les orientalistes le construisent - où le reconstruisent.

\section{L'orientalisme : un projet commun et trois} niveaux

La thèse de Said est simple: l'Orient que peignent les orientalistes, les hommes qu'ils y décrivent, les sociétés 
qu'ils y analysent, les religions qu'ils y observent, sont faits pour répondre aux questionnements, aux inquiétudes, à l'anxiété des Occidentaux face à une réalité qu'ils ne connaissent pas et qui leur paraît à la fois attirante et menaçante.

L'orientalisme, qui a une longue histoire, est unifié par un projet commun : celui de construire l'Orient pour répondre aux questionnements de l'Occident. Il s'est développé à plusieurs niveaux : il analyse de manière scientifique une certaine aire, avec ses sociétés et ses cultures; il saisit parallèlement ce qui différencie de manière radicale cette entité de l'Occident, et l'appréhende dans un imaginaire projeté par les orientalistes :

"Par orientalisme, j'entends plusieurs choses qui, à mon avis, dépendent l'une de l'autre. L'acception la plus généralement admise de ce terme est universitaire $[\ldots]$

"A cette première tradition universitaire $[\ldots]$ se rattache une conception plus large de l'orientalisme: style de pensée fondé sur la distinction ontologique et épistémologique entre 'l'Orient' et [...] 'l'Occident" (Said, 1980/1978, p. 1).
"Il y a un échange incessant entre l'orientalisme au sens universitaire et l'orientalisme de l'imaginaire [...]" (Said, 1980/1978, p. 15).

Au-delà de ces acceptions, une autre existe aussi, qui n'est ni académique, ni imaginaire :

"J'en arrive ainsi au troisième sens de l'orientalisme qui est défini de manière plus historique et plus matérielle que les deux autres. Prenant comme point de départ, très grossièrement, la fin $\mathrm{du}$ XVIII ${ }^{\mathrm{e}}$ siècle, on peut décrire et analyser l'orientalisme comme l'institution globale qui traite de l'Orient, qui en traite par des déclarations, des prises de position, des descriptions, un enseignement, une administration, un gouvernement" (Said, 1980/1978, p. 15).

C'est cette troisième composante de l'orientalisme qui est au coeur du propos de Said, car c'est elle qui transforme une spéculation intellectuelle en outil de domination :

$$
\begin{aligned}
& \text { "Bref, l'orientalisme est un } \\
& \text { style occidental de }
\end{aligned}
$$


domination,

de

restructuration et d'autorité sur l'Orient. La notion de discours développée par

Michel Foucault dans l'Archéologie du savoir et Surveiller et punir m'a servi à caractériser l'orientalisme" (Said, 1980/1978, p. 14-15).

Comment passe-t-on en effet d'une forme de connaissance à un outil de domination ? C'est le point essentiel de la thèse de Said. Pour l'établir, il mobilise à la fois son métier d'historien de la littérature et la réflexion contemporaine sur les discours, les lieux où ils sont formulés et la forme qu'ils revêtent : c'est de Foucault (et un peu de Barthes) que vient, en particulier, cette partie de son inspiration.

\section{Une analyse littéraire de la genèse d'un courant de pensée}

Said donne toute la mesure de son immense talent de comparatiste dans l'admirable galerie de portraits d'orientalistes qu'il dresse et qui lui permet de suivre l'évolution des idées qui les fédèrent. Il y a ceux qui formalisent les premières traditions, comme Barthélémy d'Herbelot à la fin du XVII ${ }^{\mathrm{e}}$ siècle, ceux qui ESPAÇO E CULTURA, UERJ, RJ, N. 34, P.17-50, JUL./DEZ. DE 2013 http://www.e-publicacoes.uerj.br/index.php/espacoecultura/ siècle, tels Antoine Silvestre de Sacy ou Ernest Renan, ceux qui le parent, par leurs récits de voyages ou leurs souvenirs, d'une dimension littéraire, Edward William Lane, René de Chateaubriand, Alphonse de Lamartine, Gustave Flaubert, Gérard de Nerval, ceux qui le mettent en œuvre pour asseoir la domination coloniale, Lord Cromer ou Lord Curzon, ceux qui en tirent parti pour manipuler le monde arabe, tels Lawrence d'Arabie, ou ceux qui, des années 1920 au début des années 1960, portent à sa perfection l'orientalisme académique et ses utilisations politiques, Louis Massignon en France, Sir Gibb en Grande-Bretagne puis aux Etats-Unis.

Said souligne l'originalité de chacun de ces orientalistes et de sa démarche : pour rapporter un témoignage utile sur l'Egypte, Lane se fait passer pour un indigène grâce à la complicité d'un ami : il rentre ainsi dans l'intimité d'un peuple qui lui serait sans cela resté étranger, mais il n'y parvient qu'en se censurant, qu'en sacrifiant sa personnalité. A l'opposé, l'itinéraire qui mène Chateaubriand jusqu'à Jérusalem est moins fait pour parler d'Orient que pour mettre perpétuellement en valeur l'égo de l'illustre écrivain. Said rend sensible la profonde sympathie que Flaubert éprouve pour un Orient dont les couleurs le ravissent, et 
dont il découvre avec passion la sensualité. Le plus grand et le plus singulier de ces orientalistes, c'est peut-être Louis Massignon : une érudition éblouissante, un catholicisme qui le conduit à privilégier la perspective religieuse, l'islam - mais que curieusement, il appréhende surtout dans ses formes mystiques, chez les soufis, ou chez Mansour al-Hallaj, dont le comportement christique le fascine - il finit crucifié.

Said rend sensible la spécificité, la profondeur ou la superficialité de tous ces auteurs. Il les analyse longuement, car il y voit des acteurs essentiels de la construction et de l'évolution de l'orientalisme :

"Je me sépare de Michel Foucault, à l'œuvre de qui je dois beaucoup, sur un point, écrit-il : je crois en l'influence déterminante d'écrivains individuels sur le corpus des textes, par ailleurs collectif et anonyme, constituant une formation discursive telle que l'orientalisme" (Said, 1980/1978, p. 37).

De sa formation de littéraire, Said a gardé le goût du détail concret, dont il connaît la valeur :

"Ce qui m’intéresse le plus, comme homme de science, ce n'est pas la vérité politique brute, mais le détail, de même que ce qui nous intéresse chez quelqu'un comme Lane, ou Flaubert, ou Renan, ce n'est pas la vérité indiscutable (pour lui) que les Occidentaux sont supérieurs aux Orientaux, mais le témoignage fortement élaboré et modulé qu'en donnent les détails de son œuvre à l'intérieur du très vaste espace frayé par cette vérité" (Said, 1980/1978, p. 27-28).

L'intérêt que le lecteur porte à l'ouvrage de Said tient à l'évocation de ces hommes et de ces œuvres sans lesquels l'orientalisme n'existerait pas; il résulte de la fraîcheur et de la finesse des analyses qu'il en propose. On y sent la sensibilité à vif d'un Palestinien révolté par le mépris dans lequel l'Arabe et le musulman sont tenus par beaucoup de ces spécialistes de l’Orient.

\section{De l'œurre individuelle au discours}

La liberté du génie créateur, que souligne Said, a cependant ses limites :

"[...] nous cultivons une mythologie de la création : nous croyons que le génie 
artistique, un talent original ou un intellect puissant peut franchir d'un bond les limites de sa propre époque pour proposer au monde une œuvre nouvelle. Il y a une part de vérité dans des idées comme celle-ci, on ne peut le nier. Néanmoins, les possibilités de travail qui se présentent dans la culture à un esprit original ne sont jamais illimitées; il est vrai qu'un grand talent a un respect sain pour ce que les autres ont fait avant lui et ce que son domaine renferme déjà. L'œuvre de ceux qui l'ont précédé, la vie institutionnelle d'un domaine scientifique, la nature collective de toute entreprise savante : tout cela, sans parler de la situation économique et sociale, a tendance à limiter la portée de la production personnelle du savant. Un domaine comme l'orientalisme a une identité cumulative et collective, une identité particulièrement forte étant donné qu'il est associé avec la science traditionnelle (les classiques, la Bible, la philologie), les institutions

publiques

(gouvernements, compagnies

commerciales, sociétés géographiques, universités) et des écrits déterminés par leur genre (récits de voyages, d'exploration, fictions, descriptions exotiques). Cela a donné une sorte de consensus : certaines choses, certains types d'affirmations, certains types d'ouvrages ont paru corrects pour l'orientaliste" (Said, 1980/1978, p. 232-233).

Les orientations de l'orientalisme sont définies ou exprimées par quelques grands esprits, mais l'œuvre de ceux-ci s'insère dans une tradition et dans un milieu qui pèsent sur eux. Elle reflète une position visà-vis de l'objet 'Orient' qui est également contraignante. C'est ainsi que débute la transformation de positions individuelles en formation discursive :

"[L'orientalisme] est un discours qui n'est pas du tout en relation de correspondance directe avec le pouvoir politique brut, mais qui, plutôt, est produit et existe au cours d'un échange inégal avec différentes sortes de 


\begin{abstract}
pouvoirs, qui est formé jusqu'à un certain point par l'échange avec le pouvoir politique (comme dans l'establishment colonial ou impérial), avec le pouvoir intellectuel (comme dans les sciences régnantes comme la linguistique, l'anatomie comparée, ou l'une quelconque des sciences politiques modernes), avec le pouvoir culturel (comme dans les orthodoxies et les canons qui régissent le goût, les valeurs, les textes), la puissance morale (comme dans les idées que 'nous' faisons et de ce qu'ils' ne peuvent faire ou comprendre comme nous). En fait ma thèse est que l'orientalisme est - et non seulement représente - une dimension considérable de la culture politique et intellectuelle moderne et que, comme tel, il a moins de rapports avec l'Orient qu'avec 'notre' monde" (Said, 1980/1978, p. 25).
\end{abstract}

On passe ainsi de la vue individuelle, avec ses fulgurances, à l'énoncé formaté - et détaché de ses référents!
Comme discours, l'orientalisme est un produit culturel, qui participe à la logique générale des systèmes culturels. Pour analyser celle-ci, Said s'appuie sur Gramsci et sur l'opposition que celui-ci établit entre société civile et société politique. La culture fait partie de la première :

"La culture, bien sûr, fonctionne dans le cadre de la société civile, où l'influence des idées, des institutions et des personnes s'exerce non par la domination mais par ce que Gramsci appelle le consensus. Dans une société qui n'est pas totalitaire, certaines formes culturelles prédominent donc sur d'autres, tout comme certaines idées sont plus répandues que d'autres: la forme que prend cette suprématie est appelée hégémonie par Gramsci, concept indispensable pour comprendre quelque chose à la vie culturelle de l'Occident industriel. C'est l'hégémonie, ou plutôt les effets de l'hégémonie culturelle, qui donne à l'orientalisme la constance et la force dont j'ai parlé" (Said, 1980/1978, p. 19). 
De la masse des publications sort, par une sorte d'effet mécanique, la transformation des positions individuelles en une 'formation discursive' :

"De plus tout ouvrage sur l'Orient s'associe à d'autres ouvrages, à des publics, à des institutions, à l'Orient luimême. L'ensemble des relations entre les ouvrages, les publics et certains aspects de l'Orient constitue donc une formation analysable $[\ldots]$ dont la présence dans le temps, dans le discours, dans les institutions (écoles, bibliothèques, bureaux des affaires étrangères) lui donne force et autorité" (Said, 1980/1978, p. 34).

Dès l'instant où une connaissance est organisée en discours, une logique de la répétition se met en place; elle finit par dessiner l'objet sur lequel porte l'énonciation sans qu'il lui soit nécessaire de ressembler au réel :

"La géographie imaginaire [...] légitime un vocabulaire, un univers $d u$ discours représentatif particulier à la discussion et à la compréhension de l'islam et le l'Orient. Ce que ce discours considère comme un fait - par exemple que Mahomet est un imposteur est une composante du discours, une assertion que le discours oblige à prononcer chaque fois que le nom Mahomet apparaît. Soustendant les différentes unités du discours orientaliste $[\ldots]$, il y a un ensemble de figures représentatives, ou tropes. Ces figures sont à l'Orient réel [...] ce que les costumes sont aux personnages d'une pièce [...]. Autrement dit, nous n'avons pas besoin de chercher une correspondance entre le langage utilisé pour dépeindre l'Orient et l'Orient lui-même; ce n'est pas parce que ce langage est imprécis, mais parce qu'il ne cherche même pas à être précis. Ce qu'il tente de faire, c'est [...] du même coup à caractériser l'Orient comme étranger et à lui donner corps schématiquement sur la scène d'un théâtre dont le public, le directeur et les acteurs sont pour l'Europe et seulement pour l'Europe. D'où l'oscillation entre le familier et l'étrange: Mahomet est 
toujours l'imposteur [...] et

toujours l'Oriental" (Said,

1980/1978, p. 88-89).

L'orientalisme devient ainsi un discours détaché des réalités, et soumis à des contraintes qui le façonnent en profondeur :

"Mes principales hypothèses de travail ont été et sont toujours les suivantes: les domaines scientifiques, tout autant que les œuvres de l'artiste, même le plus original, subissent des contraintes et des pressions extérieures et des influences stabilisatrices: écoles, bibliothèques,

gouvernements ; en outre, les écrits, qu'ils soient érudits ou de fiction, ne sont jamais libres, mais sont limités dans leur jeu d'images, leur présupposés et leurs intentions; et enfin, les progrès faits par une 'science' comme l'orientalisme sous sa forme universitaire sont moins objectivement vrais que nous n'aimons souvent à le croire. Bref, j'ai tenté jusqu'ici, dans mon travail, de décrire l'économie qui fait de l'orientalisme une discipline ESPAÇO E CULTURA, UERJ, RJ, N. 34, P.17-50, JUL./DEZ. DE 2013 cohérente, même en admettant que, comme idée, comme concept ou comme image, le mot Orient a une grande résonnance culturelle en Occident" (Said, 1980/1978, p. 232).

De l'analyse individuelle, on est passé au discours institué.

\section{L'orientalisme comme fondement d'un projet politique}

Comme Said le souligne, il existe un troisième niveau d'orientalisme : l'orientalisme comme "institution globale, qui traite de l'Orient, qui en traite par des déclarations, des prises de position, des descriptions, un enseignement, une administration, un gouvernement" (p. 14) Comment passe-t-on des deux premiers niveaux (le texte académique et le discours) au troisième?

Pour Said, le discours finit par imprégner totalement la conscience de ceux qui sont en rapport avec l'Orient. A ce sujet, il propose de distinguer "entre une positivité presque inconsciente (et certainement intouchable), qu' [il] appellera l'orientalisme latent, et les différentes affirmations sur la société, les langues, les littératures, l'histoire, la sociologie, etc. 
qu'[il] appellera l'orientalisme manifeste" (p. 236). L'orientalisme latent, produit à long terme du discours orientaliste, est générateur d'attitudes et de comportements stéréotypés à l'égard de l'oriental, de l'Arabe, du musulman, ou ailleurs, du Persan, de l'Hindou - ce qui caractérise précisément l'orientalisme manifeste.

Une mutation se produit :

"Nous devons tenir compte
d'un long et lent processus
d'appropriation par lequel
l'Europe, ou plutôt la
conscience européenne de
l'Orient, se transforme : [à
l'origine], elle était textuelle
et contemplative. Elle devient
administrative, économique
et même militaire. La
transformation fondamentale
a été d'ordre spatial et
géographique, ou, plutôt,
c'est la qualité de
l'appréhension spatiale et
géographique qui s'est
transformée dans la mesure
où il était question de
l'Orient" (Said, 1980/1978, p.
7 ).

La familiarité plus grande qui naît de la facilité nouvelle des déplacements change la signification du discours orientaliste : il n'est plus simplement porteur d'une affirmation de supériorité ; il justifie et facilite l'entreprise impérialiste.

"Le fait de désigner, depuis des siècles, l'espace géographique situé à l'Est de l'Europe par le terme 'd'oriental' relevait pour une part de la politique, pour une part de la doctrine et pour une part de l'imaginaire: il n'impliquait pas de lien nécessaire entre l'expérience authentique de l'Orient et la connaissance de ce qui est oriental" (Said, 1980/1978, p. 241)

Cette situation change avec le progrès des communications :

"Sous l'en-tête général de la connaissance de l'Orient, et sous le parapluie de l'hégémonie occidentale, à partir de la fin du XVIII ${ }^{\mathrm{e}}$ siècle, a émergé un Orient complexe, bien adapté aux études académiques, aux expositions dans les musées, à la reconstruction par les bureaux coloniaux, à l'illustration théorique des thèses anthropologiques, biologiques, linguistiques, 
raciales et historiques sur

l'humanité et l'univers, par

exemple des théories

économiques

et

sociologiques sur le

développement, la révolution,

la personnalité culturelle, le

caractère national ou

religieux. De surcroît, la prise

en compte par l'imagination

des choses de l'Orient était

plus ou moins exclusivement

fondée sur une conscience

occidentale souveraine; de sa

position centrale indiscutée

émergeait un monde oriental

conforme d'abord aux idées

générales de ce qu'était un

Oriental, puis à une logique

détaillée gouvernée non

seulement par la réalité

empirique, mais par toute une

batterie de désirs, de

répressions,

d'investissements et de

projections"

(Said,

1980/1978, p. 20).

Avec des voyages plus rapides et plus sûrs, les contacts se multiplient: l'orientalisme change de signification :

"Quand Lane, Renan, Burton et les centaines de voyageurs et de savants européens au
XIX $^{\mathrm{e}}$ siècle parlent de

l'Orient, nous pouvons immédiatement remarquer une attitude bien plus intime, et même possessive, envers l'Orient et les choses de l'Orient. Sous la forme classique et souvent éloignée dans le temps dans lequel il était reconstruit par l'orientaliste, sous la forme précisément réelle où il était vécu, étudié, imaginé, l'espace géographique de l'Orient était pénétré, travaillé, fortement saisi. L'effet cumulatif de décennies d'un traitement si souverain de la part de l'Occident a fait passer l'Orient d'un espace étranger à un espace colonial " (Said, 1980/1978, p. 242).

On passe d'une simple spéculation à une doctrine partagée par des institutions qui pénètrent l'espace et induisent sa colonisation.

Voici, rapidement résumées, l'articulation des divers sens de l'orientalisme, et la manière dont il finit par déboucher sur la prise en mains de l'Orient par l'Occident : 
"La relation entre l'Occident et l'Orient est une relation de pouvoir et de domination: l'Occident a exercé à des degrés divers une hégémonie complexe $[\ldots]$. L'Orient a été orientalisé non seulement parce qu'on a découvert qu'il était 'oriental' selon les stéréotypes de l'Européen moyen du XIX ${ }^{\mathrm{e}}$ siècle, mais encore parce qu'il pouvait être rendu oriental" (Said, 1980/1978, p. 18).

Une relation de pouvoir et de domination ? Une relation politique, donc :

"Ce que je prétends, c'est que l'orientalisme est fondamentalement une doctrine politique imposée à l'Orient parce que celui-ci était plus faible que l'Occident, qui supprimait la différence de l'Orient en la fondant dans sa faiblesse" (Said, 1980/1978, p. 234).

Une situation durable, des contextes changeants.

\section{L'orientalisme : une structure perceptuelle engendrée par la situation}

L'orientalisme tel que l'analyse Said est un curieux mixte de continuité et de changement. Il apparaît d'abord comme une structure générale de la perception qui donne au phénomène son unité à travers les siècles - et les millénaires. Cette structure n'évolue pas, car elle reflète une situation qui nait des interrogations et des inquiétudes que suscite le monde étrange et étranger qui s'étend à l'Est de l'Europe, et de la position qu'occupent ceux qui répondent aux questions ainsi posées :

"Pour l'Occident, l'Asie
autrefois représentait la
distance muette et
l'étrangeté : l'islam était
l'hostilité militante envers la
chrétienté européenne. Pour
surmonter ces constantes
redoutables, l'Orient demandait à être connu, puis envahi et conquis, puis recréé par des savants, des soldats, des juges qui avaient déterré des histoires, des races et des cultures oubliées pour les avancer - au-delà de ce que l'Oriental moderne connaissait lui-même - en tant que véritable Orient classique, qui put être utilisé pour juger et gouverner l'Orient moderne. Un Orient 
de serre chaude apparaissait ;

le mot Orient était un

vocable d'érudit, qui

désignait ce que l'Europe

moderne venait de faire d'un

Est encore original" (Said,

1980/1978, p. 110-111).

Mais si la situation qui donne à l'opposition Occident/Orient sa dimension ontologique ne change pas, la manière dont elle s’applique évolue :

"Ce que je tente de montrer ici, c'est que la transition entre une appréhension, une formulation ou une définition purement et simplement textuelles et leur mise en pratique s'est produite, et que l'orientalisme a joué un grand rôle dans cette transition [...]. Dans la mesure où il était question du travail strictement érudit de l'orientalisme $[\ldots]$, celui-ci a beaucoup à son actif. Pendant sa grande époque, au $\mathrm{XIX}^{\mathrm{e}}$ siècle, il a produit des érudits, il accru le nombre des langues enseignées en Occident et la quantité de manuscrits édités, traduits et commentés; dans bien des cas, il a fourni à l'Orient des étudiants européens pleins de sympathie, qui s'intéressaient réellement à des sujets tels que la grammaire sanscrite, la numismatique phénicienne et la poésie arabe. Cependant et il nous faut parler ici très clairement -, l'orientalisme a dominé l'Orient. En tant que système de pensée sur l'Orient, il s'est toujours élevé du détail spécifiquement humain au détail général 'transhumain' : une observation sur un poète arabe $\mathrm{du} \mathrm{X}^{\mathrm{e}}$ siècle se multipliait d'elle-même pour devenir une politique envers (et à propos de) la mentalité orientale en Egypte, en Iran et en Arabie. De même un vers du Coran pouvait être considéré comme la meilleure preuve d'une sensualité musulmane indéracinable. L'orientalisme supposait un Orient immuable, absolument différent de l'Occident (les raisons pour cela changent d'époque à époque). Et l'orientalisme, sous la forme qu'il a prise après le XVIII ${ }^{e}$ siècle, n’a jamais pu se revoir et se corriger lui-même. Tout cela rend inévitable Cromer 
et Balfour, comme

observateurs

et

administrateurs de l'Orient"

(Said, 1980/1978, p. 115).

Cette disposition est présente en Grèce classique. Elle informe toute la relation de la chrétienté médiévale et renaissante avec le monde musulman; elle perdure au XIX $^{\mathrm{e}}$ siècle. Anouar Abdel Malek, que cite Said, la résume admirablement :

"Sur le plan de la position $d u$ problème, de la problématique, [les orientalistes] considèrent l'Orient et les Orientaux comme 'objet' d'étude frappé d'altérité - comme tout ce qui est autre, qu'il soit 'sujet' ou 'objet' - mais d'une altérité constitutive, de caractère essentialiste [...]. Cet 'objet' d'étude sera, comme il se doit, passif, non participant, doté d'une subjectivité 'historique' pardessus tout, non actif, non autonome, non souverain par devers soi : le seul Orient ou Oriental ou 'sujet' qu'on pourrait admettre, à l'extrême limite, est l'être aliéné, philosophiquement, c'est-à-dire autre que luimême par rapport à luimême, posé, entendu, défini - et agi - par autrui [...]" (Abdel Malek, cité par Said, 1980/1978, p. 116).

Abel Malek en tire une conclusion générale, qui est aussi celle de Said :

"On aboutit ainsi à une typologie [...] qui fait de 'l'objet' étudié un être autre par rapport auquel le sujet est transcendant [...]. On voit combien, du XVIII ${ }^{\mathrm{e}}$ siècle au $\mathrm{XX}^{\mathrm{e}}$ siècle, l'hégémonisme des minorités possédantes, mis à nu par Marx et Engels, et l'anthropocentrisme démantelé par Freud s'accompagnent de l'européocentrisme en matière de sciences humaines et sociales, et plus particulièrement dans celles en rapport direct avec les peuples non européens" (Abdel Malek, cité par Said, 1980/1978 p. 116-117).

Cette façon de concevoir l'autre demeure stable, parce qu'elle reflète une situation qui ne se modifie pas. Cela n'empêche cependant pas l'évolution des 
perspectives retenues. Dans ce domaine, une grande coupure se produit à la fin du $\mathrm{XVIII}^{\mathrm{e}}$ siècle.

\section{Les formulations longtemps religieuses de} l'orientalisme

Jusqu'aux Lumières, la question que pose l'Orient est celle de la religion: le christianisme est né au Proche-Orient, en Palestine ; il y rompt avec la foi hébraïque et la prolonge à la fois; ses racines s'étendent à la Mésopotamie d'Ur et des patriarches, à l'Egypte de Moïse, à la Babylone de la captivité - elles se confondent à la fois avec le Proche et avec le Moyen-Orient. Les chrétiens ne peuvent se désintéresser d'un ensemble de terres où s'est constituée leur foi : leur histoire les lie aux pays du Levant, au minimum à une portion de ceux-ci : la Terre Sainte.

L'islam est le troisième grand monothéisme issu de la tradition d'Abraham. Comment un chrétien peut-il accepter qu'une religion née six siècles après la venue du Christ se prétende la 'vraie' religion? Il ne peut s'agir que d'une erreur. La tentation est donc grande de voir dans l'Islam une imposture et de ne pas reconnaître à l'Oriental la même qualité d'être, la même authenticité qu'à l'Occidental.

La présentation de l'Oriental comme un être inférieur aurait dû disparaître au moment où la confrontation religieuse islam/chrétienté cesse d'être au premier plan. Il n'en est rien: les questions que soulève ce 'non-sens' historique ont continué - et continuent - à soulever l'étonnement, témoin Claude Lévi-Strauss comparant dans Tristes Tropiques bouddhisme, islam et christianisme :

"On ne saurait imaginer de contraste plus marqué que celui du Sage [Bouddha] et du Prophète [Mahomet]. Ni l'un ni l'autre ne sont des dieux, voilà leur unique point commun. A tous les autres égards, ils s'opposent [...]. Mais aussi 1200 ans les séparent; et c'est le malheur de la conscience occidentale que le christianisme, qui, né plus tard, eût pu opérer leur synthèse, soit apparu 'avant la lettre', non comme une conciliation a posteriori de deux extrêmes, mais passage de l'un à l'autre: terme moyen d'une série destinée par sa logique interne, par la géographie et par l'histoire à se développer désormais dans 
le sens de l'Islam; puisque ce dernier - les Musulmans triomphent sur ce point représente la forme la plus évoluée de la pensée religieuse, sans pour autant être la meilleure; je dirais même, en étant, pour cette raison la plus inquiétante des trois" (Lévi-Strauss, 1955/1965, p. 368).

\section{La laïcisation de l'orientalisme depuis l'époque des Lumières}

Les questions que les Occidentaux se posent au XVIII ${ }^{\mathrm{e}}$ siècle changent. Jusqu'alors :

"Lorsque le mot Orient n'était pas pris simplement comme le synonyme de l'Est asiatique dans son ensemble, ou pour désigner de manière générale le lointain et l'exotique, il était compris de manière tout à fait rigoureuse comme s'appliquant à l'Orient islamique" (Said, 1980/1978, p. 92-93).
La curiosité pour l'Orient cesse alors d'être fondamentalement religieuse : sa dominante devient laïque.

Les voyages, les textes et les monuments soulignent désormais la splendeur des cultures qui ont laissé leur empreinte sur cette partie du monde; le présent n'offre rien d'équivalent. Quelle leçon l'Occident peut-il en tirer ? Pourquoi l'Orient actuel ne revêt-il pas la même grandeur? Pourquoi ne pèse-t-il pas davantage dans le domaine de la science et de la philosophie? Pourquoi ses arts apparaissent-ils comme figés ? Tout ceci témoigne d'un déclin: au Proche- et au Moyen-Orient, celui-ci n'est-il pas imputable à l'islam ?

Dans le même temps, maintenant que les voyages sont plus faciles et les contacts plus fréquents, l'image de l'Orient change: il fascine ceux qui le connaissent par ses couleurs (elles enchantent les peintres que tente l'orientalisme) et par sa sensualité.

L'idée que les civilisations de l'Orient sont en retard sur celles de l'Occident dans une course au progrès dont les Lumières ont fait la théorie reproduit, dans une sphère profane, celle qui résultait de l'interprétation des rapports entre christianisme et islam. Ce qui est plus nouveau, c'est la conviction que l'Orient 
offre une sensibilité et des richesses spirituelles qui manquent à l'Occident. Said insiste surtout sur la première perspective, mais n'ignore pas la seconde, comme le montre ce qu'il dit de voyageurs comme Flaubert ou de savants comme Louis Massignon.

L'Islam était perçu comme une fausse religion; il est devenu synonyme de déclin. Mais ainsi désacralisé et réduit à ses dimensions matérielles, à ses couleurs, à ses voluptés, il fait rêver. C'est un objet désirable. Pourquoi ne pas le conquérir? C'est ce à quoi mène l'orientalisme sous sa troisième forme, institutionnelle.

\section{Perspectives critiques sur l'Orientalisme}

L'ouvrage d'Edward Said marque un tournant dans la façon de présenter les rapports de l'Occident et du reste du monde : jamais la responsabilité de ses élites intellectuelles dans la genèse de l'impérialisme n'avait été aussi fortement soulignée.

La démonstration proposée par Said est-elle, en tout point, convaincante? Sa portée nous paraît limitée par certaines ambiguiités, par certains des partis retenus. Ces faiblesses n'invalident pas la thèse centrale de Said, la dénonciation des rapports inégaux que l'Occident entretient depuis des siècles avec l'Orient, mais elles conduisent à nuancer la vision qu'il donne des responsabilités de l'intelligence occidentale.

La thèse de Said repose sur deux idées-forces. 1- L'orientalisme naît d'une structure relationnelle relativement stable, mais constitue un ensemble qui évolue. 2L'orientalisme s'analyse en trois niveaux: un champ de questionnement et d'investigation, un discours tenu par l'intelligence occidentale, et à partir d'un certain moment, un ensemble de pratiques et de stratégies qui donnent à ceux qui les mettent en ouvre un pouvoir de domination. Le mouvement en trois temps qui a conféré à l'orientalisme ses formes actuelles est-il assez précisément analysé ? Les liens qui rendent automatique le passage entre les trois niveaux sont-ils aussi solides que Said ne le suppose ?

\section{Le ressort profond de l'orientalisme}

Ce qui est à l'origine de l'orientalisme selon Said, c'est un questionnement: celui d'hommes et de femmes qui résident au Ponant et s'interrogent sur le Levant ; celui-ci ne peut 
leur rester indifférent, car il a en partie façonné leurs façons d'être, leurs religions et leur civilisation; dans le même temps, les Occidentaux ont de la peine à comprendre les réactions des peuples d'Orient et les perçoivent comme menaçants.

Le rapport dont naît l'orientalisme s'inscrit dans ce cadre général, mais il évolue. Pour les anciens Grecs, il résulte de la conscience qu'ils ont des éléments de civilisation que le monde oriental leur a apportés, des dieux qu'ils lui ont empruntés et de la menace qu'il fait peser sur leur indépendance. Pour l'Occident devenu chrétien, il tient à ce que la foi qu'il partage est née dans une Terre Sainte aujourd'hui dominée par un islam qu’ils considèrent comme une fausse religion, et qui s'est montré plusieurs fois conquérant. Pour les Européens à partir des Lumières, l'Orient est le lieu où a germé la Civilisation, d'où elle a migré vers l'Ouest, et qu'elle a aujourd'hui étrangement déserté.

Les Grecs anciens devaient une partie de leurs mythes et de leurs techniques aux peuples qui vivaient à l'Est et au Sud, Phéniciens ou Egyptiens pour ne citer que les plus importants. Au Moyen Age, les chrétiens savent que la Révélation a pris place hors de l'espace où réside l'immense majorité d'entre eux. La conscience que les Européens modernes ont d'eux-mêmes repose sur l'idée que la civilisation dont ils incarnent aujourd'hui les formes les plus hautes résulte d'un processus qui a embrassé l'humanité entière : un part essentielle de leur histoire, celle des origines de leur culture, se situe en marge de l'aire où ils sont aujourd'hui installés.

Ceux qui essaient de répondre à ces questionnements changent au fur et à mesure que les perspectives évoluent : dans l'Antiquité grecque, les poètes et les auteurs de tragédie, qui mettent en forme une religion sans écriture et sans théologiens, incarnent la conscience grecque. Dans le monde chrétien, les spécialistes des textes sacrés se trouvent directement confrontés aux problèmes qui résultent de la localisation lointaine des lieux où a eu lieu la Révélation. Ils essaient de répondre aux questions que pose ainsi l'Orient à travers les textes anciens qui sont à leur disposition, et grâce à ce que pèlerins, commerçants ou missions diplomatiques rapportent de leurs voyages.

A partir du XVIII ${ }^{\mathrm{e}}$ siècle, c'est l'Occident en tant que civilisation qui est en jeu, les origines de celle-ci se situant ailleurs. C'est aux spécialistes des langues orientales et des textes anciens - qui sont en train de devenir des orientalistes - que l'on demande de répondre aux questions 
qui se posent ainsi, mais à une époque où les voyages deviennent plus faciles, le nombre de ceux qui ont une expérience directe de l'Orient ne cesse de croître, et les connaissances s'enrichissent d'observations directes, qui donnent une dimension ethnographique nouvelle aux savoirs sur l'Orient.

Aux trois époques, Grèce ancienne, Europe chrétienne, Europe moderne, l'Occident a besoin de l'Orient pour savoir ce dont il est parti et ce qu'il est devenu. Il $\mathrm{y}$ a derrière tout l'orientalisme une même curiosité fondamentale pour des espaces dont l'Occident a hérité une partie de ses savoir-faire, de ses idées et de sa foi; l'intérêt va davantage à ceux qui habitaient autrefois ces contrées, et dont sont venues des leçons essentielles, qu'à ceux qui les peuplent aujourd'hui, et qui apparaissent souvent comme inégaux à leurs prédécesseurs et fréquemment hostiles ou menaçants.

La thèse de Said, c'est qu'au gré d'une dérive qui fait passer des idées aux discours, et de ceux-ci aux pratiques politiques, l'évolution contemporaine finit par faire oublier la motivation première de la quête d'Orient: à l'interrogation sur l'origine de l'Occident - commune en partie à celui-ci et à l'Orient - se substitue une entreprise de conquête que justifie la supériorité de l’Europe.

Lorsqu'il évoque la grande aventure de Bonaparte en Egypte, Martin Bernal dit son admiration pour l'ouvrage de Said, mais s'en démarque sur un point :

\section{"L’Expédition [d’Egypte]} marque un tournant fascinant dans l'attitude des Européens vis-à-vis de l'Orient. Les relevés très élaborés, les cartes, les dessins ainsi que le vol des objets et des monuments destinés à embellir la France sont autant de pratiques reflétant déjà une manière d'étudier, d'objectiver par le biais de l'enquête scientifique, qui allait bientôt devenir la marque de l'impérialisme européen et l'un des fondements de 'l'orientalisme' si bien décrit par Edward Said. Il demeurait pourtant de nombreuses traces de l'attitude plus traditionnelle à l'égard de l'Egypte. Les savants de l'expédition croyaient qu'ils allaient trouver en Egypte tout ce qu'ils cherchaient à savoir sur le monde en général et leur propre culture 
en particulier, et pas

seulement dévoiler des

curiosités qui viendraient

compléter le savoir et la

domination des Occidentaux

sur l'Afrique et l'Asie"

(Bernal, 1996/1987, p. 228)

Il existait donc chez ceux qui s'embarquent pour l'Egypte autre chose qu'un désir de domination; Bernal parle à ce sujet 'd'une attitude plus traditionnelle'. Pour lui, la dette de l'Occident vis-à-vis de l'Egypte avait été longtemps reconnue par 'l'Ancien Modèle', que le philhellénisme et l'européocentrisme remettent en cause à la fin du XVIII ${ }^{\mathrm{e}}$ siècle. Mais il note aussi que ce qui motive alors les curiosités est un nouveau questionnement sur les origines de la civilisation occidentale. On le voit particulièrement à l'œuvre chez Charles François Dupuis, auteur en 1795 d'un ouvrage très remarqué sur l'Origine de tous les cultes :

"Que Dupuis ait participé directement ou non à la décision de Bonaparte de partir pour l'Egypte, la présence dans l'expédition d'une figure intellectuelle et politique comme la sienne reflète le courant d'égyptophilie qui traversait les milieux napoléoniens avant 1798, date de lancement de la Grande Expédition. On sait qu’il est responsable de la percée vers la Haute-Egypte qu'il considérait comme la source de la culture égyptienne et par conséquent mondiale" (Bernal, 1996/1987, p. 227) (c'est nous qui soulignons).

L'Orientalisme naît d'une curiosité sincère pour des contrées où sont nées les formes supérieures de la religion, puis de la civilisation. La mécanique de transformation des idées en discours, et de ceux-ci en pratiques dominatrices, est-elle suffisante pour faire totalement oublier ces attitudes? Nous ne le pensons pas - Said non plus d'ailleurs lorsqu'il écrit que "dans bien des cas, [l'orientalisme] a fourni à l'Orient des étudiants européens pleins de sympathie, qui s'intéressaient réellement à des sujets tels que la grammaire sanscrite, la numismatique phénicienne et la poésie arabe" (Said, 1980/1978, p. 115).

\section{La transformation des témoignages en discours, et de ceux-ci en pratiques}

Pour Said, la composante authentiquement désintéressée de 
l'orientalisme est effacée par la mécanique qui transforme en formation discursive les témoignages textuels et les leçons de l'anthropologie, de plus en plus nombreux maintenant que les voyages sont plus rapides et plus sûrs. L'occultation des curiosités premières est alors complétée par la mutation qui conduit aux attitudes et pratiques impérialistes.

Said s'appuie, pour analyser la première étape, sur les travaux de Foucault. Il ne peut cependant s'empêcher de les nuancer: la personnalité de ceux qui interprètent les textes anciens, produisent des écrits nouveaux ou qui font part de leur expérience personnelle compte beaucoup dans le processus; c'est à travers leur peinture de l'Orient que celui-ci prend consistance, qu'il devient une réalité parlante. C'est d'eux que viennent les thèmes qui sont tôt ou tard repris par un discours qui devient anonyme, et dans lequel ils ont puisé, et puisent, d'ailleurs une partie de leur inspiration première. Il n'y aurait pas de formation discursive s'il n'y avait pas de créateurs; ceux-ci n'existent d'autre part que parce qu'ils sont capables de s'affranchir au moins partiellement des récits et textes narratifs qui ont contribué à les former. Les idées se transforment en permanence en discours plus ou moins formatés, mais de manière tout aussi permanente, les créateurs remettent en cause ces grands récits. Le structurationnisme avant l'heure que pratique Said limite la portée de sa thèse : oui, les travaux des orientalistes débouchent sur des formations discursives, mais celles-ci sont constamment reprises, retouchées ou remises en cause par ceux qui travaillent sur les textes anciens, voyagent ou font des séjours ethnographiques dans les aires concernées. Le passage des idées nouvelles aux grands récits est dialectique.

Le second processus quanalyse Said, c'est celui qui transforme le discours en pratiques et règles d'action, et fait de la description des faiblesses de l'Orient un instrument de domination. Ceux qui travaillent sur les textes ou tirent parti de leurs travaux ethnographiques, et ceux qui les lisent et adoptent en les simplifiant leurs manières de voir, ne sont pas les seuls en cause. La transformation n'est possible, Said le souligne, que parce que l'Orient est devenu plus proche et qu'il est parcouru par un nombre croissant d'Occidentaux. Est-ce du discours orientaliste, qui les imprègne sans doute, qu'ils tirent leur sentiment de supériorité, leur arrogance et leur volonté de domination? Les thèmes orientalistes peuvent justifier leurs attitudes, mais ils ne les génèrent pas: les marins, les commerçants, les missionnaires, les 
voyageurs qui parcourent l'Orient font directement l'expérience de la supériorité que leur confèrent la qualité de leurs moyens de communications et de transport, de certaines de leurs techniques et de leurs armes, comme l'efficacité de leurs systèmes juridiques. Dans la mesure où leur attitude suscite des réactions et un rejet parfois violent de leur présence, ils réclament, pour les protéger et les aider, l'intervention de l'Etat dont ils sont citoyens, ou celle du concert des puissances européennes, comme on le voit en Chine ou en Turquie.

Dans son schéma d'interprétation, l'importance que Said attache à la pénétration sans cesse plus poussée du monde oriental par des Occidentaux suggère que la responsabilité des orientalistes demeure limitée: ce ne sont pas eux qui révèlent aux Européens leur supériorité; les voyageurs qui font l'expérience des espaces extra-européens n'ont pas besoin de théoriciens pour prendre la mesure des possibilités d'action dont ils jouissent en Orient ou dans d'autres régions. L'orientalisme reflète cette situation: il traite d'espaces où la pénétration de l'Occident commence : c'est ce qui explique les contours flexibles de l'aire qu'il analyse. A la suite des progrès de la navigation européenne, il incorpore l'Asie méridionale et orientale. Dans la mesure où existent là des civilisations de l'écrit, avec des textes anciens, l'orientalisme savant y découvre un nouveau champ - mais il se complète de plus en plus par des savoirs de type ethnographiques. En Afrique subsaharienne, dans la plus grande partie des Amériques et en Océanie, dont les sociétés ne pratiquent que l'oralité, ces derniers sont les seuls à compter.

Ce que dit Said de l'attitude des colonisateurs dans des pays à vieille culture savante, monde musulman, Inde ou Chine, il pourrait le dire aussi des terres qui n'ont jamais connu de civilisations de l'écrit : là aussi, les colonisateurs tirent parti de la production savante européenne ethnographique évidemment - pour justifier leur domination, mais celle-ci résulte d'abord des rapports inégaux que leur capacité de voyager, de communiquer et de se battre fait naître.

La seconde étape du processus qu'analyse Said pour instruire le procès de l'orientalisme n'est pas responsable de l'extension de la domination européenne, même s'il lui fournit des justifications et certains moyens d'action. Le discours 'orientaliste' n'apparaît pas comme un préalable à l'extension de l'impérialisme son rôle est, tout au plus, de justification.

La situation contemporaine le montre. Les empires européens ont disparu. 
Les discours dominants sont anticoloniaux et anti-impérialistes. Placées sous l'égide de l'ONU, des Etats européens, de l'Europe unie, des Etats-Unis, du Japon et aujourd'hui de la Chine, les formes d'aide internationale se multiplient. Les intentions des ONG sont pures, mais leurs membres se trouvent, comme c'était le cas au XIX siècle, dans des situations inégales vis-à-vis des populations auxquelles ils portent secours. Ils disposent de richesses ou de bien que tous convoitent autour d'eux; ils ont des moyens de communication et de transports efficaces. Certains ne résistent pas à la tentation de profiter de leur situation pour s'enrichir, ou abuser de filles et de garçons dont ils n'auraient jamais obtenu les faveurs chez eux. Certains touristes profitent de même des situations inégales que leur offrent les pays en voie de développement. Des comportements 'impérialistes' sont présents dans des sociétés qui les condamnent explicitement.

Que l'orientalisme ait servi de justification à l'entreprise coloniale, qu'il lui ait fourni des instruments indispensables à l'affirmation de son emprise, c'est exact, mais la mécanique de transformation des idées en formations discursives, et de cellesci en schèmes et pratiques de domination, qu'invoque Said - avec une certaine prudence - n’explique pas la pénétration européenne. Celle-ci s'appuie d'abord et surtout sur les avantages que ses techniques de transport et de communication, et ses armements, lui procuraient au $\mathrm{XIX}^{\mathrm{e}}$ siècle, et assurent aujourd'hui encore aux Européens, aux Américains, aux Japonais ou aux Chinois lorsqu'ils vivent ou voyagent hors de chez eux.

\section{Les diverses structures spatiales de l'orientalisme}

L'Orient de l'orientalisme dont traite Said est une réalité géographique, mais une réalité floue. Est-elle homogène? $\mathrm{Ne}$ conviendrait-il pas plutôt de parler d'une pluralité d'Orients et d'orientalismes ? Dans la mesure où l'Orient de l'orientalisme classique répond aux inquiétudes, aux craintes et aux aspirations de l'Occident, ne reflète-t-il pas aussi les contradictions et les problèmes de l'Occident lui-même - c'est une question que Said n’aborde pas.

Les Occidentaux ne portent pas le même regard sur toutes les terres qui s'étendent au Levant: certaines les intéressent intellectuellement et émotivement plus que d'autres, parce qu'ils situent là l'origine de ce à quoi ils croient, des techniques qu'ils utilisent et des savoirs 
qu'ils mobilisent. Les Grecs anciens s'attachent ainsi plus particulièrement à l'Egypte et à la Phénicie, alors qu'ils craignent la Perse, mais ne s'en sentent pas redevables. Les chrétiens centrent leur Orient sur Nazareth, Bethleem et Jérusalem, et l'étendent à l'ensemble de la Terre Sainte et des territoires adjacents. C'est l'islam, né à la Mecque et à Médine, qu'ils redoutent.

Avec la laïcisation du regard porté sur l'Orient, la vision géographique qu'en donne l'Ouest change et revêt plusieurs configurations selon les moments et selon les groupes. Pour la franc-maçonnerie, dont le développement coïncide avec le passage d'une vision religieuse à une vision laïque $d u$ Levant, l'Egypte, avec ses mystères, ses hiéroglyphes, ses temples, ses pyramides et les connaissances géométriques qui leur sont liées, est la terre des origines, celle qu'il importe de connaître.

Avec les Lumières, l'histoire de l'humanité s'allonge: pour comprendre ce qu'elle est, il convient d'en retracer les premiers pas. Rousseau témoigne de ce mouvement. Il importe pour lui de saisir comment les hommes sont passés de la pureté de l'état de nature à la civilisation et à ses vices :

"En découvrant et suivant ainsi les routes oubliées et perdues qui de l'état naturel ont dû mener l'homme à l'état civil; en rétablissant, avec les positions intermédiaires que je viens de marquer, celles que le temps qui me presse m'a fait supprimer, ou que l'imagination ne m'a point suggérées, tout lecteur attentif ne pourra qu'être frappé par l'espace immense qui sépare ces deux états. C'est dans cette lente succession des choses qu'il verra la solution d'une infinité de problèmes de morale et de politique, que les philosophes ne peuvent résoudre. Il sentira que le genre humain d'un âge n'était pas le genre humain d'un autre âge" (Rousseau, 1755, II).

Pour comprendre l'homme moderne, il est de la plus haute importance de reconstituer "cette lente succession des choses". Comment y parvenir? En observant l'homme là où les transitions sont encore visibles : en voyageant. Les récits dont nous disposons proviennent malheureusement d'observateurs grossiers. Il faudrait que les voyageurs se fassent philosophes : 
"Supposons un Montesquieu, un Buffon, un Diderot, un Duclos, un d'Alembert, un Condillac, ou des hommes de cette trempe, voyageant pour instruire leurs compatriotes, observant et décrivant, comme ils savent faire, la Turquie, l'Egypte, la Barbarie [...], le pays des Cafres, l'intérieur de l'Afrique $[\ldots]$, les royaumes de Siam, de Pégu et d'Ava, la Chine [...]; sans oublier l'autre hémisphère, le Mexique, le Pérou $[\ldots]$, s'il était possible enfin les Caraïbes, la Floride et toutes les contrées sauvages; voyage le plus important de tous et celui qu'il faudrait faire avec le plus de soin: supposons que ces nouveaux Hercules, de retour de ces courses mémorables, fissent ensuite à loisir l'histoire naturelle, morale et politique de ce qu'ils auraient vu, nous verrions nous même sortir un monde nouveau de dessous leur plume, et nous apprendrions ainsi à connaître le nôtre" (Rousseau, 1755, Note X).
Le voyage philosophique, qui trouve ici sa justification, et qu'illustrent bientôt les périples de James Cook et de Bougainville, retrace la marche de la civilisation. Il fait une place de choix à l'histoire des peuples dotés d'écriture. A la veille de la Révolution, le récit du voyage de Volney (1787) en Egypte, Palestine, Syrie, en fournit l'exemple le plus connu.

(i) Plusieurs cartes mentales du processus de civilisation émergent de ces réflexions. La plus fréquente, celle qui est la plus directement liée à l'idée de progrès, s'affirme à la fin du XVIII ${ }^{e}$ siècle et connaît un succès considérable au début du XIX ${ }^{\mathrm{e}}$ : la marche de l'histoire s'inscrirait dans un mouvement de très longue durée; il aurait fait migrer les foyers d'innovation de l'Asie vers l'Europe et les conduirait aujourd'hui en Amérique. Le progrès revêt ainsi une double inscription temporelle et géographique. Il a commencé en Chine ou aux Indes, a balayé le Moyen-Orient Perse, Mésopotamie, Syrie, Egypte -, puis a touché la Grèce, Rome et enfin l'Europe occidentale et du Nord. L'Afrique subsaharienne, qui ignore l'écriture, témoigne encore de ce qu'était l'humanité primitive, comme le souligne Hegel (1979), le plus illustre représentant de ce courant. C'est de l'orientalisme ainsi compris et délimité que parle l'ouvrage d'Edward Said. 
(ii) Mais au sein de l'Orient dilaté et balayé par les premières formes du progrès, toutes les terres ne se valent pas. La tradition grecque et chrétienne le centrait sur l’Egypte, la Phénicie ou la Terre Sainte. Les Lumières emboîtent le pas. Les avancées de la philologie modifient la donne :

"Nous avons déjà fait le lien entre la famille des langues sémitiques établie par Schölzer et les travaux de l’abbé Barthélémy, ainsi que les recherches conduites à Göttingen. En 1820, des savants, dont le Danois Christian Rask et le disciple de Herder, Franz Bopp, avaient recherché systématiquement les liens unissant la phonétique et la morphologie de la plupart des langues européennes.

"Cette entreprise avait un rapport évident avec la nouvelle taxonomie ou classement systématique des races. Puisque les Caucasiens venaient des montagnes d'Asie, on supposait que les langues européennes avaient la même origine. Puisque les Allemands étaient censés avoir quitté en dernier

l'Urheimat, la terre d'origine, ils étaient nécessairement les Caucasiens les plus purs et l'allemand devenait plus pur et plus ancien que les autres langues de la même famille. D'où le terme allemand pour désigner cette famille de langues récemment définie: Indogermanisch [...]. Le mot est dû à l'indianiste allemand H. J. Klaporth en 1823. Franz Bopp, quant à lui, préférait pourtant le terme utilisé par les savants des autres pays, 'indo-européen', apparu en 1816 sous la plume de Thomas Young" (Bernal, 1996/1987, p. 278).

Deux images de l'Orient se développent alors chez les spécialistes : celle qui met l'accent sur le rôle des peuples sémites, installés en Arabie, en Mésopotamie, en Syrie, au Liban et en Palestine, et celle qui s'attache aux peuples Aryens, Indiens, Perses Mèdes et Hittites. Ceux qui appartiennent à ce second groupe minimisent le rôle des peuples sémites et de l'Egypte. Pour eux, l'Occident n'est pleinement lui-même que là où se sont imposées des races supérieures - en Grèce et en Allemagne. 
L'idée d'une qualité particulière des Allemands et de leur langue est ancienne elle remonte à la Réforme :

"Au cours de ces mêmes
décennies, les
prenaient d'analogies frappantes entre leur langue et le grec. [...] Le lien entre les deux langues se trouva renforcé après la Réforme et la rupture avec le catholicisme 'romain', et une nouvelle image s'imposa : celle du grec et de l'allemand comme les deux langues du protestantisme. C'est en retournant au Nouveau Testament grec que Luther fourbit ses armes contre l'Eglise de Rome. Le grec devenait une langue chrétienne sacrée et les protestants avaient de bonnes raisons de la présenter comme plus authentiquement chrétienne que le latin" (Bernal, 1996/1987, p. 238).

Le thème prend une nouvelle consistance au XVIII ${ }^{\mathrm{e}}$ siècle, à un moment où l'Allemagne divisée doute de son génie :

"Les romantiques allemands [...] tentèrent de faire retrouver aux Allemands leurs racines culturelles et de créer une civilisation allemande authentique sur le sol et sur le peuple allemand. Les nouvelles idées romantiques et progressistes exigeaient que l'on présentât désormais les peuples dans leur contexte géographique et historique. Le génie de la race ou l'esprit appartenant à une terre et à son peuple changeaient de forme selon l'esprit de l'époque, [...] selon son Zeitgeist. Un peuple conservait cependant toujours son essence immuable. La figure la plus éminente de cet aspect du mouvement romantique, Johann Gottfried Herder, joua aussi un rôle important dans le développement du néo-hellénisme et de la linguistique" (Bernal, 1996/1987, p. 253).

C'est dans ce cadre que s'impose l'image d'un Orient supérieur par sa pureté, même s'il est peuplé de peuples cavaliers aux mœurs rudes, et dont les techniques demeurent plus frustes dans beaucoup de domaines que celles de l'Egypte ou de la 
Mésopotamie - un Orient dont les qualités se retrouvent dans l'Occident germanique, et plus généralement, dans l'Europe du Nord, et assure sa supériorité :

"Il y avait $[\ldots]$ cette tendance de plus en plus affirmée, venue $\mathrm{du}$ romantisme allemand, et qui situait les origines de l'humanité - et par conséquent celles des Européens - dans les régions montagneuses de l'Orient et non pas dans les vallées du Nil et de l'Euphrate, comme le croyaient les Anciens. A la recherche de l'homme primitif, Herder jugeait nécessaire 'd'escalader péniblement les montagnes jusqu'au toit du monde, en Asie'.

"Herder situait les origines de l'homme dans l'Himalaya. Dans l'ensemble, les romantiques, jusqu’à la fin du $\mathrm{XIX}^{\mathrm{e}}$ siècle, estimèrent que l'humanité, et en particulier ses représentants les plus 'purs', les Aryens, venaient des hauts-plateaux de l'Asie. L'un des avantages de cette version 'asiatique' des origines était de situer les Allemands plus près que les
Européens de l'Ouest des sources pures de l'humanité [...]" (Bernal, 1996/1987, p. 271).

L'orientalisme n'est pas seulement une entreprise de dénigrement systématique des peuples qui vivent au Levant. C'est un moyen de hiérarchiser les peuples d'Occident.

\section{L'exclusion de l'orientalisme allemand est-} elle justifiée?

Certains choix d'Edward Said étonnent : celui d'avoir limité l'analyse de l'orientalisme à ses composantes française, anglaise et américaine. Pourquoi ne pas avoir fait une place à l'orientalisme allemand ? Said sait qu'on peut le critiquer sur ce point et justifie son parti :

"Mon étude peut présenter un aspect trompeur : à part quelques références occasionnelles, je n'étudie pas de manière exhaustive les progrès faits par les savants allemands après la période inaugurale dominée par Silvestre de Sacy [...].

"L'œuvre de l'érudition allemande a été de raffiner et 
de perfectionner des

techniques s'appliquant à des textes, à des mythes, à des idées, à des langues recueillis presque littéralement en Orient par l'Angleterre et la France impériales (Said, 1980/1978, p. 32).

La raison que Said donne de ce choix? L'Allemagne n'étant devenue que tardivement une puissance coloniale, ses orientalistes n'ont pas eu l'opportunité de développer toutes les facettes de leur discipline, celles qui sont le plus directement liée à l'impérialisme en particulier :

"Du point de vue de l'histoire et de la culture, il y a une différence aussi bien quantitative que qualitative entre l'engagement francobritannique en Orient et [...] celui de toutes les autres puissances européennes et atlantiques. Par conséquent, parler de l'orientalisme, c'est parler essentiellement, mais non exclusivement, d'une entreprise de civilisation anglaise et française $[\ldots] "$ (Said, 1980/1978, p. 16)

Said explicite plus loin l'argument :
"Or, parce que la GrandeBretagne, la France et maintenant les Etats-Unis sont des puissances impériales, leur société politique communique à leur société civile un sens de l'urgence, une imprégnation politique directe, pourrait-on dire, partout et chaque fois que des questions se rapportant à leurs intérêts impériaux à l'étranger sont en jeu. Je pense par exemple que l'on peut dire $[\ldots]$ qu'en s'intéressant à l'Inde ou à l'Egypte, un Anglais de la fin $\mathrm{du}$ XIX $^{\mathrm{e}}$ siècle ne perdait jamais de vue le fait qu'il s'agissait de colonies britanniques" (Said, 1980/1978, p. 23-24).

L'argument est-il convaincant ? Non: l'Allemagne unifiée ne se contente pas de créer des colonies en Afrique tropicale (Cameroun, Kenya, Tanganyika, Sud-Ouest africain) ou en Océanie. Elle s'intéresse à l'Orient et pénètre l'Empire ottoman, comme le manifeste la construction du chemin de fer vers Bagdad, le Baghdadbahn.

L'exclusion de l'orientalisme allemand - et des autres orientalismes 
européens - est choquante pour une autre raison. $\mathrm{Au} \mathrm{XIX}^{\mathrm{e}}$ siècle et au début $\mathrm{du} \mathrm{XX}^{\mathrm{e}}$, le mélange d'attirance et de mépris à l'égard l'Orient dont traite Said n'est pas propre à la France et à la Grande-Bretagne. La perspective orientaliste est partagée par tous les pays. Par leurs commerçants ou par leurs missionnaires, tous les peuples européens participent à la pénétration et à la domination des espaces non européens, même s'ils n'en prennent pas le contrôle. Des cohortes d'Occidentaux de tous les pays prennent part à la projection universelle de l'Europe et s'appuient sur la France, sur 1"Angleterre ou sur le concert des nations civilisées, comme en Chine.

Dans ces conditions, exclure l'orientalisme allemand est d'autant plus gênant que son autorité morale est plus grande :

"Cependant, ce que
l'orientalisme allemand a eu
de commun avec
l'orientalisme anglo-français,
puis l'orientalisme américain,
c'est une espèce d'autorité
sur l'Orient à l'intérieur de la
culture occidentale" (Said,
1980/1978, p. 32-33).

Si l'orientalisme justifie la pénétration occidentale en Orient, c'est parce qu'il se présente comme un savoir digne de respect : c'est à sa version allemande qu'il le doit largement.

Négliger l'orientalisme allemand, c'est enfin renoncer à l'analyse de ce que l'orientalisme nous apprend sur l'Occident lui-même, sur sa diversité, sur les clivages qui le caractérisent, sur les hiérarchies qui y existent et les justifications qui en sont données.

\section{Conclusion}

L'Orientalisme est un grand livre. On y lit la curiosité que l'Occident manifeste à l'égard des pays du Levant ; on y voit la diversité de ceux qui construisent l'image de ces pays encore lointains. La connaissance intime que Said a de leurs œuvres et la sympathie qu'il manifeste à l'égard de certains d'entre eux rendent passionnante la lecture de l'ouvrage.

L'Orient dont parle Said n'est pas une réalité naturelle : c'est une construction historique. Les géographes sont séduits par la démonstration qu'il en propose.

Said tire parti des travaux des années 1960 sur les formations discursives pour montrer comment un ensemble de contributions individuelles se fond en un grand récit, qui pèse à son tour sur les chercheurs, mêmes les plus originaux, et 
aboutit à une interprétation simplifiée et tendancieuse d'un monde dont la pénétration s’accélère.

Selon Said, l'évolution va plus loin chez les peuples impérialistes, Français ou Britanniques. Le discours orientaliste y donne naissance à des convictions qui justifient la prise en main de pays déclinants et incapables de tirer parti de leurs richesses; il est à l'origine des attitudes qu'endossent les nouveaux arrivés, et des recettes qui facilitent la tâche des marins, des militaires et des administrateurs qui assurent la conquête des nouvelles colonies et les 'mettent en valeur'.

Alors que sa démarche structurationniste conduisait Said à aborder avec une certaine prudence le passage des œuvres individuelles aux formations discursives, la manière dont il restitue la seconde étape du processus, la transition qui mène $d u$ discours aux pratiques dominantes, apparaît plus fragile. Said indique, bien sûr, que la transformation qu'il analyse est liée avec la familiarité croissante des Occidentaux avec des espaces de plus en plus faciles à atteindre et à visiter, mais sans insister sur l'expérience directe de la domination que leur assurent leur supériorité technique et les doctrines juridiques qu'ils professent.
L'accent mis sur le caractère global de l'orientalisme conduit Said à négliger la diversité des images géographiques que l'orientalisme construit à partir de la fin du $\mathrm{XVIII}^{\mathrm{e}}$ siècle, et à passer sous silence le rôle qu'elles jouent dans la conception même que les Occidentaux se font de leur propre civilisation.

Said retrace de manière passionnante la genèse les images des pays du Levant que l'orientalisme fournit à l'Occident. Il a raison de dénoncer leurs biais systématiques et la manière dont elles ont servi à justifier la pénétration impérialiste. Mais lorsqu'il essaie de mener sa démonstration un pas plus loin et prétend établir que l'orientalisme pousse l'Europe à la conquête du monde, son analyse paraît moins fondée, car elle ne fait pas assez de place à l'expérience directe de l'inégalité que réservent les contacts avec des peuples techniquement moins équipés.

Soucieux de mettre l'accent sur le passage du discours à la domination, Said simplifie la lecture géographique qu'il propose de l'orientalisme. Il néglige la diversité des images que celui-ci construit, et la hiérarchisation qu'elle établit au sein des Orientaux, comme aussi des Occidentaux. 
* Université de Paris IV-Sorbonne

Référence

BERNAL, M., 1996/1987, Black Athena. Le racines afroasiatiques de la civilisation classique, vol. 1, L'Invetion de la Grèce antique, Paris, PUF; éd. original américaine, Black Athena, vol. 1, The Fabrication of Ancient Greece (1785-1985), New Brunswick (N. J.), Rutgers University Press

HEGEL, G.F.K, 1979, La Raison dans I'histoire. Introduction à la philosophie de l'histoire, Paris, 10/18. Trad. de l'éd. allemande de 1955, d'après les cours et les notes de Hegel 1822-1828.

LÉVI-STRAUSS, C., 1965/1955, Tristes Tropiques, Paris, Plon ; cité d'après l'édition 1965.

ROUSSEAU, J.-J., 1755, Discours sur l'origine de l'inégalité, Paris. SAID, E. W., 1980/1978, L'Orientalisme. L'Orient vu parzl'Occident, Paris, le Seuil; éd. or. américaine, Orientalism, New York, Pantheon Book; Londres, Routledge et Kegan Paul.

SAID, E. W., 2000/1993, Culture et impérialisme, Paris, Fayard ; éd. or. américaine, Culture and imperialism, New York, Knopf, Londres, Chatto et Windus.

VOLNEY (C. F. Chassebeuf, dit), 1787, Voyage en Syrie et en Egypte durant les années 1783, 1784 et 1785, Paris.

UMA RELEITURA DE EDWARD SAID

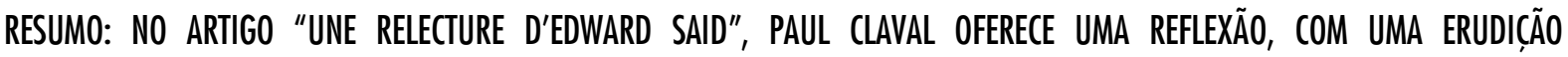
DESLUMBRANTE, SOBRE 0 ORIENTALISMO DIFUNDIDO NA OBRA DE SAID E SOBRE OS SEUS DESDOBRAMENTOS NO MUNDO CONTEMPORÂNEO. 0 ORIENTALISMO, SEGUNDO SAID, CONSTITUI UM DISCURSO SOBRE 0 ORIENTE, ENQUANTO CONSTRUÇÃO MENTAL, QUE LEGITIMA PROCESSO DE DOMINAÇÃO EUROPEIA SOBRE ESSE TERRITÓRIO. PAUL CLAVAL RESSALTA A IMPORTÂNCIA DA OBRA DE SAID NO QUE DIZ RESPEITO À CONSTRUÇÃO HISTÓRICA DAS ENTIDADES GEOGRÁFICAS ORIENTE E OCIDENTE. CONTUDO, ELE APONTA AS FRAGILIDADES OU LIMITES DAS INTERPRETAÇ̃̃ES DE SAID SOBRE 0 ORIENTALISMO. EDWARD SAID NEGLICENCIA A DIVERSIDADE DE IMAGENS QUE $O$ ORIENTALISMO CONSTRUIU E NÃO ABORDA AS CONTRADIÇÕES E OS PROBLEMAS DO PRÓPRIO OCIDENTE. PARA CLAVAL, NÃO HÁ APENAS UM ORIENTE, UM ORIENTALISMO, MAS MÚLTIPLOS ORIENTES E MÚLTIPLOS ORIENTALISMOS. HÁ DIVERSAS GEOGRAFIAS IMAGINÁRIAS QUE PERMITEM A CONSTRUC̦̃̃O E A COMPREENSÃO DAS RELAÇ̃̃ES ENTRE 0 ORIENTE E 0 OCIDENTE, E 0 RESTO DO MUNDO.

PALAVRA-CHAVE: EDWARD SAID - ORIENTALISMO - GEOGRAFIAS IMAGINÁRIAS - DISCURSO 
A REREADING EDWARD SAID

ABSTRACT: IN THE ARTICLE "D'UNE RELECTURE EDWARD SAID," PAUL CLAVAL OFFERS A REFLECTION ABOUT THE ORIENTALISM DIFFUSED OVER THE WORK OF SAID AND ABOUT THEIR DEVELOPMENTS IN THE CONTEMPORANY WORLD. THE ORIENTALISM BY SAID, IS A DISCOURSE ON THE EAST, AS A MENTAL CONSTRUCTION, WHICH LEGITIMIZES THE CASE OF EUROPEAN DOMINATION ON THAT TERRITORY. PAUL CLAVAL EMPHASIZES THE IMPORTANCE OF WORK OF SAID WITH REGARD TO THE HISTORICAL CONSTRUCTION OF THE GEOGRAPHICAL ENTITY OF EAST AND WEST. HOWEVER, CLAVAL ALSO STRESS THE WEAKNESSES OR LIMITS OF SAID'S INTERPRETATIONS ON ORIENTALISM. FOR HIM, SAID NEGLECTS THE DIVERSITY OF IMAGES THAT BUILT ORIENTALISM AND DOES NOT ADDRESS THE CONTRADICTIONS AND OWN PROBLEMS OF THE WEST. FOR CLAVAL, THERE IS NOT ONLY ONE EAST, OR AN ORIENTALISM, BUT MULTIPLE EASTS AND MULTIPLE ORIENTALISMS. THERE ARE MANY IMAGINARY GEOGRAPHIES THAT ALLOWS THE CONSTRUCTION AND UNDERSTANDING OF THE RELATIONSHIP BETWEEN THE EAST AND THE WEST, AND THE REST OF THE WORLD.

KEYWORD: EDWARD SAID - ORIENTALISM - GEOGRAPHIES IMAGINARY - DISCOURSE 